\title{
Una reseña de Trastornos pragmáticos
}

\section{A review of Pragmatic Disorders}

\author{
Carlos Álvarez E. \\ Departamento de Fonoaudiología \\ Universidad de Chile
}

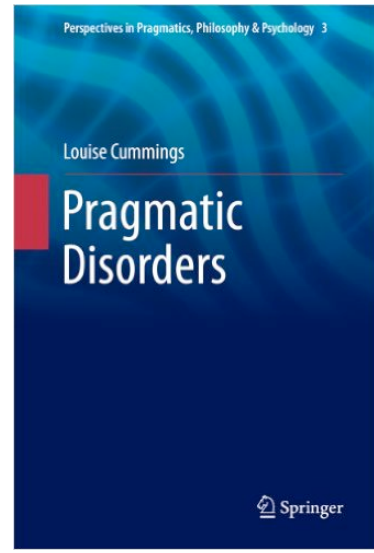

Autor: Louise Cummings

Editorial: Dordrecht, Springer

Número de páginas: XIII + 255

Año: 2014

ISBN: 978-94-007-7954-9

Datos autor: Profesora e investigadora en School of Arts \& Humanities, Nottingham Trent University, Inglaterra.

En el tercer volumen de la serie Perspectives in Pragmatics, Philosophy \& Psychology de la editorial Springer, Louise Cummings nos presenta un valioso trabajo académico que tiene por objeto dar cuenta del estado del arte de una subdisciplina que, en las últimas décadas, ha experimentado un considerable desarrollo teórico-práctico en relación con las alteraciones que pueden afectar la competencia pragmática de un ser humano. Esta subdisciplina se denomina pragmática clínica. 
Al comienzo de esta obra, la autora argumenta que el desarrollo de la pragmática clínica se sustenta en dos pilares fundamentales: el primero se relaciona con el crecimiento y consolidación de la pragmática como disciplina lingüística; mientras que el segundo, con la inminente necesidad de desarrollar formas efectivas de evaluación e intervención clínicas en el ámbito pragmático. En los siete capítulos de esta obra, se enfatiza la necesidad de establecer un vínculo cada vez más cercano entre estos dos pilares fundamentales para poder desarrollar terapias efectivas y de base científica, que ayuden a personas con trastornos pragmáticos de variada naturaleza.

A continuación, se presenta una breve descripción de los contenidos de cada uno de los apartados que componen la obra.

El primer capítulo, titulado Pragmatics and Language Pathology, entrega al lector los fundamentos teórico-prácticos sobre los cuales se sustenta la pragmática clínica. La autora comienza el capítulo definiendo ocho aspectos centrales del ámbito de la pragmática y el discurso: actos de habla, implicatura, presuposición, deixis, lenguaje no literal, contexto, cohesión discursiva y coherencia discursiva. Estos conceptos son definidos y ejemplificados con claridad y precisión teórica. También son ilustrados mediante interacciones en las cuales participan personas con trastornos pragmáticos asociados con patologías tales como afasia de Broca, afasia de Wernicke, síndrome de Asperger y complejo de demencia asociado a VIH, entre otros. La vinculación entre las definiciones teóricas y las interacciones antes señaladas constituye una primera aproximación para entregar al lector una visión integrada entre los aspectos propios de la pragmática lingüística y su vinculación con el quehacer clínico.

Pragmatic Disorders Across the Life Span constituye el segundo capítulo de esta obra. En este se presenta al lector una revisión en profundidad de los trastornos pragmáticos asociados a diversas patologías factibles de observar en cuatro estadios del ciclo vital del ser humano: developmental period (0-7 años), older childhood and adolescence (8-17 años), early to late adulthood (18-65 años) y advanced adulthood (66-85 años). Al respecto, en el primer estadio, se examinan los trastornos pragmáticos asociados con el Trastorno Específico del Lenguaje, Trastorno de Espectro Autista, Trastornos Emocionales y Conductuales y Discapacidad Intelectual. En el segundo estadio, se da cuenta de los trastornos pragmáticos relacionados con tumores cerebrales y lesiones cerebrales traumáticas. En el tercer estadio, los trastornos pragmáticos son analizados considerando su vínculo con la esquizofrenia, lesiones cerebrales traumáticas y trastornos neurodegenerativos. Finalmente, en el cuarto estadio, se exponen los trastornos pragmáticos que pueden ocasionar un daño en el hemisferio cerebral izquierdo, un daño en el hemisferio cerebral derecho y en las demencias. 
El siguiente capítulo de la obra, Disorders of the Pragmatics-Cognition Interface, se examina la función de habilidades cognitivas, tales como memoria y atención, en la interpretación de enunciados en actos comunicativos. Se inicia con un tratamiento detallado de la relación entre pragmática y cognición. El desarrollo temático se efectúa analizando, desde una perspectiva pragmática, un intercambio comunicativo de dos turnos. Los fenómenos descritos son implicatura, presuposición, actos de habla y deixis. Asimismo, se presenta un análisis de los procesos cognitivos implicados, a saber atención y percepción, en el intercambio comunicativo. Se establece con claridad la estrecha relación entre estos procesos y los fenómenos pragmáticos ya analizados. Además, se trata con profundidad la relación entre dos aspectos cognitivos, la teoría de la Mente y las funciones ejecutivas, y el desarrollo típico de la competencia pragmática y sus posibles alteraciones clínicas. Se describen detalladamente los fundamentos teóricos de cada uno de estos aspectos, mencionando investigaciones relevantes que respaldan de manera empírica su rol en el desarrollo pragmático.

Theoretical Models and Pragmatics, cuarto capítulo del libro, expone la influencia que han tenido tres modelos teóricos de base cognitiva en el trabajo clínico en relación con los trastornos pragmáticos. Se describen y analizan, críticamente, tres teorías que han sido ampliamente utilizadas en contextos clínicos y han posibilitado conocer en profundidad las características del desempeño pragmático de pacientes con algún trastorno clínico. Las teorías analizadas son: teoría de la relevancia de Sperber y Wison, teoría pragmática cognitiva de Bara y teoría de la pragmática modular de Karshen.

El quinto capítulo del texto, The Impact of Pragmatic Disorders, entrega al lector un análisis profundo del efecto que tiene en la vida de niños y adultos la presencia de un trastorno pragmático. Se presenta un análisis de cómo los ámbitos psicológico, social, académico, ocupacional y vocacional, conductual y forense se ven afectados. El análisis de cada uno de estos ámbitos se sustenta en recientes investigaciones realizadas en variados contextos y mediante diferentes aproximaciones metodológicas.

Pragmatic Disorders in Complex and Underserved Populations constituye el sexto capítulo de la obra. Se dedica de manera especial a indagar acerca de la presencia de trastornos pragmáticos en tres poblaciones a las cuales no se les ha brindado la suficiente atención clínica en el ámbito comunicacional: niños con trastornos emocionales y conductuales, adolescentes y adultos privados de libertad en centros penitenciarios y adultos con demencias no asociadas a la enfermedad de Alzheimer. Aunque se entregan antecedentes de investigaciones relacionadas con aspectos pragmáticos deficitarios en estas poblaciones, la evidencia científica para sustentar la elaboración de 
programas terapéuticos para atenderlas clínicamente es aún escasa. Por esta razón, se incentiva al lector a realizar investigaciones con el objeto de ir en ayuda de estas personas.

La obra concluye con el capítulo siete, titulado Pragmatic Disorders and Social Communication. En él, se trata la incidencia de los trastornos pragmáticos en la comunicación social. Se presenta la comunicación social como un constructo que, sin duda, debe estar al centro de la discusión de los trastornos pragmáticos en los seres humanos. De acuerdo con ello, el capítulo examina cómo este constructo se ve afectado por trastornos pragmáticos asociados a diversas patologías clínicas. También se entrega al lector una serie de herramientas comúnmente utilizadas para la evaluación y posterior tratamiento de alteraciones en la comunicación social en niños y adultos.

Luego de la revisión antes presentada, se puede concluir que Pragmatic Disorders constituye un valioso aporte a los estudios del lenguaje y la comunicación en general, y a la pragmática clínica, en particular. Escrito con lucidez y compromiso con el desarrollo de la subdisciplina, la obra cumple ampliamente el objetivo de entregar al lector una revisión clara y profunda del estado del arte de la pragmática clínica y de incentivar la realización de estudios que permitan seguir ampliando y consolidando su quehacer clínico. Ambas cualidades hacen de esta obra una publicación de consulta obligada para estudiantes de pregrado y posgrado como profesionales de la salud cuya labor se centra en el lenguaje y la comunicación humanas. 